\title{
Transkranielle elektrische Hirnstimulation
}

\section{Transcranial Electrical Brain Stimulation}

\author{
Autoren \\ Janine Reis, Brita Fritsch \\ Institut \\ Klinik für Neurologie und Neurophysiologie, Albert-Ludwigs- \\ Universität, Freiburg

\begin{abstract}
Schlüsselwörter
nichtinvasive Hirnstimulation, Schlaganfall, Neuroplastizität, Neurorehabilitation
\end{abstract} \\ Keywords \\ noninvasive brain stimulation, stroke, neuroplasticity, \\ neurorehabilitation \\ Bibliografie \\ DOI http://dx.doi.org/10.1055/s-0042-123844 \\ Akt Neurol 2017; 44: 561-567 \\ (c) Georg Thieme Verlag KG Stuttgart · New York \\ ISSN 0302-4350 \\ Korrespondenzadresse \\ Dr. Janine Reis, MD \\ Klinik für Neurologie \\ Universitatsklinikum Freiburg \\ Breisacher Straße 64 \\ 79106 Freiburg \\ janine.reis@uniklinik-freiburg.de
}

\section{ZUSAMMENFASSUNG}

Die transkranielle elektrische Hirnstimulation durch schwachen Gleichstrom (tDCS) oder Wechselstrom (tACS, tRNS) wird klinisch und experimentell zunehmend eingesetzt, um kognitive und motorische Funktionen bei gesunden Personen und Patienten mit neurologischen Erkrankungen zu verbessern. Diese Übersichtsarbeit fokussiert auf den therapeutischen Stellenwert der Gleichstromstimulation in der Neuro-rehabilitation und bietet eine Übersicht zur Studienlage bei motorischen und nicht-motorischen Symptomen nach Schlaganfall, Aufmerksamkeits- und Bewusstseinsstörungen sowie bei Morbus Parkinson.

\section{ABSTRACT}

Transcranial electrical brain stimulation using weak direct current (tDCS) or alternating current (tACS) is being increasingly used in clinical and experimental settings to improve cognitive and motor functions in healthy subjects and neurological patients. This review focuses on the therapeutic value of transcranial direct current stimulation for neurorehabilitation and provides an overview of studies addressing motor and non-motor symptoms after stroke, disorders of attention and consciousness as well as Parkinson's disease.

\section{Hintergrund}

In den letzten 10 Jahren ist die nichtinvasive elektrische Hirnstimulation als innovatives Therapieverfahren zur Unterstützung von Neurorehabilitation zunehmend in den Fokus klinisch-wissenschaftlicher Forschung gerückt. Hierzu wird mithilfe von auf der Kopfhaut platzierten Elektroden schwacher Gleichstrom (engl. transcranial direct current stimulation, tDCS), seltener Wechselstrom (transcranial alternating current stimulation, tACS), appliziert. Üblicherweise werden bis zu $0,8 \mathrm{~A} / \mathrm{m}^{2}$ für bis zu $40 \mathrm{~min}$ in einer einzelnen Stimulationseinheit verwendet [1]. Dieser Strom durchdringt partiell die unterliegenden Strukturen und beeinflusst Nervenzellen, Glia und Gefäße im stimulierten Hirnareal [1,2]. Frühe tierexperimentelle Studien der 60er und 70er Jahre zu den Effekten schwacher Gleichstromstimulation belegten bereits eine auch nach Ende der Stimulation für mehrere Stunden andauernde Erregbarkeitsänderung von Neuronen [3,4]. Therapeutische Studienansätze der 70er Jahre, damals überwiegend zur Behandlung von Depression, erbrachten jedoch keine Erfolge, was retrospektiv betrachtet auf die

Stimulationsparameter zurückgeführt werden könnte. Erst im Jahr 2000 erwirkten die Schlüsselexperimente von Nitsche und Paulus zur polaritätsabhängigen Erregbarkeitsveränderung im motorischen System des Menschen nach trankranieller Applikation von tDCS eine Renaissance der Methode [5]. Die Autoren dokumentierten eine auch über die Stimulationsdauer hinaus erhöhte kortikale Erregbarkeit, gemessen an der Amplitude motorisch evozierter Potenziale bei gesunden Probanden, nach einer mindestens 9-minütigen anodalen Stimulation über dem Motorkortex [6]. Eine Umkehr der Stimulationsrichtung (kathodale tDCS) resultierte in einem erniedrigten motorisch evozierten Potenzial. Neben dem Konzept reiner Erregbarkeitsmodulation belegen eine Vielzahl von Arbeiten auf verschiedene Art und Weise die Modulation von Neuroplastizität durch tDCS. Diese reichen von grundlagenwissenschaftlichen und mechanistischen Erkenntnissen zur Verbesserung der synaptischen Übertragungsstärke [7-9], über langanhaltende Beeinflussung von Lernprozessen und Verhalten $[10,11]$, bis hin zum therapeutischen Ansatz zur Funktionsverbesserung bei neurologischen 
und psychiatrischen Erkrankungen, die mit einer veränderten oder gestörten Neuroplastizität einhergehen (Übersicht in [12]). Vor allem erscheint die simultane Applikation von tDCS mit unterschiedlichen Lernparadigmen, wie bspw. dem motorischen oder kognitiven Training, günstige Effekte bei Gesunden und in unterschiedlichen Patientenkollektiven hervorzurufen [11,13].

Nachfolgend werden exemplarisch die Effekte der tDCS zur Funktionsverbesserung bei einigen neurologischen Krankheitsbildern, die regelmäßig im Fokus neurorehabilitativer Behandlung stehen, dargestellt. Dazu zählt vor allem der Schlaganfall. Ergänzend dazu verweisen wir auf eine aktuelle Datenbank klinischer Studien mit umfangreicher Auflistung von wissenschaftlichen und klinischen Arbeiten zur tDCS bei neurologischen und psychiatrischen Erkrankungen [14].

\section{Motorische Störungen nach Schlaganfall}

Weltweit zählt der Schlaganfall zu den Hauptgründen für bleibende Einschränkungen von Motorik und Sprache. Trotz intensiver Rehabilitationsmaßnahmen bleiben langfristig ca. 50\% der Patienten in ihrer motorischen oder sprachlichen Fähigkeit eingeschränkt [15-17]. Das derzeitige Verständnis der Mechanismen der tDCS beruht zum Großteil auf Daten, die für das motorische System dokumentiert sind. Gründe dafür sind unter anderem das Vorhandensein direkter und gut objektivierbarer Messkriterien (z. B. motorisch evoziertes Potenzial, motorische Feinfunktion), zum anderen die anatomische Zugänglichkeit motorischer Hirnareale für die nichtinvasive Stimulation. Es ist daher nicht verwunderlich, dass dem Krankheitsbild des Schlaganfalles mit dem häufigen Symptom einer Hemiparese als „Läsionsmodell der Pyramidenbahn“ ein großes wissenschaftliches Interesse für die Untersuchung von Effekten der tDCS zuteil wurde. Dies zeigt sich in einer Vielzahl wissenschaftlicher Publikationen seit 2005 ( $\triangleright$ Abb. 1). Im Gegensatz zu frühen überwiegend mechanistischen Studien ist in den letzten 5 Jahren ein Trend hin zu Studien mit klinisch orientierter therapeutischer Fragestellung zu verzeichnen.
Nach einem Schlaganfall entsteht, bedingt durch die Hirnverletzung selbst, ein kortikales Milieu, welches neuroplastische Vorgänge begünstigt. Elektrophysiologische Daten und Ergebnisse der funktionellen Bildgebung belegen eine aktive Beteiligung beider Hemisphären (ipsiläsionell als auch kontraläsionell) an der funktionellen Erholung [18-20], mit variierender Rolle zu verschiedenen Zeitpunkten nach dem Akutereignis. Aufbauend auf dem übergeordneten Konzept einer interhemisphärischen Konkurrenz belegen die Daten bei Patienten eine schlechtere Erholung im Falle eines persistierenden funktionellen Ungleichgewichtes zwischen beiden Hemisphären (Überaktivierung der nicht-betroffenen Hemisphäre) [18]. Daraus ergibt sich die grundsätzliche Überlegung, mit tDCS die Wiederherstellung eines funktionellen Gleichgewichtes zwischen beiden Hemisphären zu erwirken, entweder durch aktivierende (anodale) Stimulation der betroffenen Hemisphäre, oder durch Aktivität unterdrückende (kathodale) Stimulation der nichtbetroffenen Hemisphäre ( $\triangleright$ Abb. 2). Diese beiden StimulationsMontagen mit dem Motorkortex als primäre Zielregion werden standardmäßig verwendet. Seit 2010 findet ein weiteres Stimulationsprotokoll Anwendung, nämlich die simultane Stimulation beider Motorkortizes (Anode ipsiläsionell, Kathode kontraläsionell). Es ist zu berücksichtigen, dass die tDCS als relativ unfokale Technik nicht nur das eigentliche Zielareal (z. B. den Motorkortex) erreicht, sondern auch andere nahegelegene Hirnareale stimuliert werden [1]. Darüber hinaus stellt das vereinfachte Konzept einer Heraufoder Herabregulierung kortikaler Funktion nur einen Richtwert dar und individuelle Faktoren mit Einfluss auf komplexe kortikale Netzwerkinteraktionen sowie der Zeitverlauf nach Schlaganfall müssen ebenfalls berücksichtigt werden.

Die aktuelle Datenlage zur Anwendung der tDCS in der Behandlung motorischer Störungen nach Schlaganfall ergibt in einer Vielzahl einzelner Arbeiten vielversprechende positive Effekte der tDCS auf die Funktion der oberen Extremität, insbesondere wenn die tDCS in Kombination mit Therapieverfahren (Physio-/Ergotherapie, CIMT, robotergestütztes Training, feinmotorisches Training) und in wiederholten Sitzungen appliziert wird [12, 21, 22]. Die Ver-

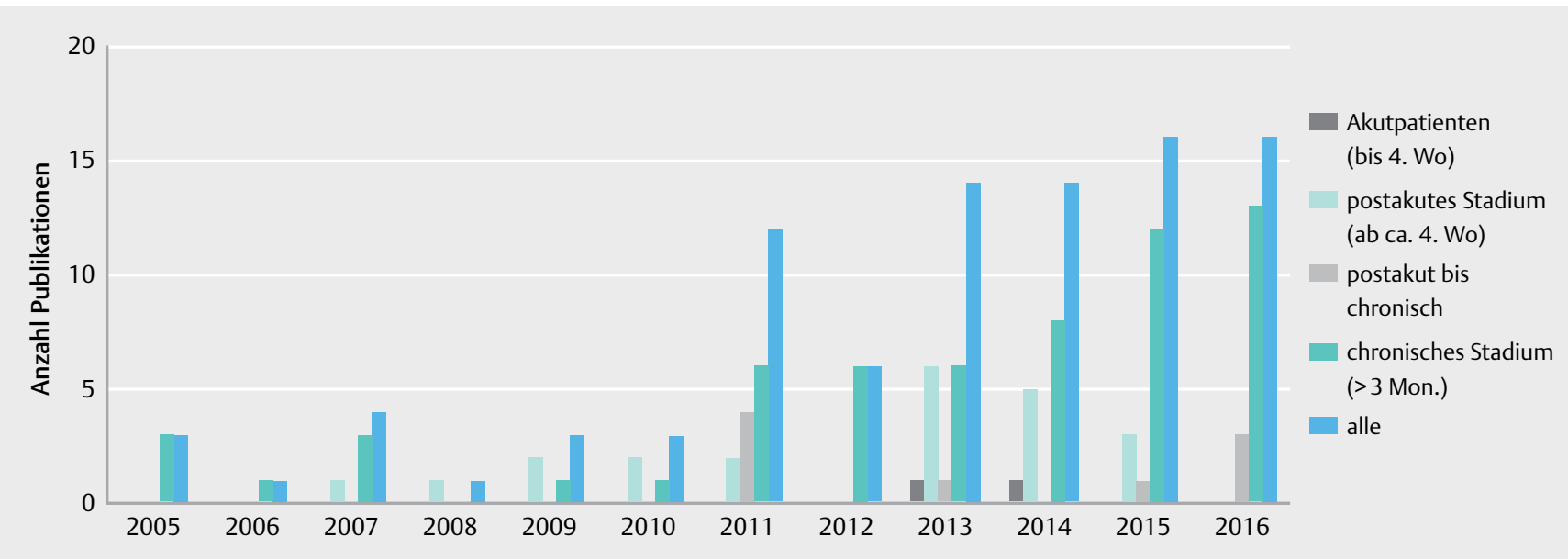

- Abb. 1 Grafische Darstellungen der publizierten Originalarbeiten pro Jahr zur tDCS bei Schlaganfallpatienten mit motorischen Symptomen. Während der größte Anteil der Studien an Patienten im chronischen Stadium ( $>3$ Monate nach Insult) durchgeführt wird, finden sich kaum Arbeiten in der Akutphase ( $<4$ Wochen, rote Balken). Dies muss bei der Beurteilung des Stellenwertes der tDCS für die Neurorehabilitation berücksichtigt werden. 
a

$$
\begin{aligned}
& \text { Aktivierung } \\
& \text { der geschädigten } \\
& \text { Hemisphäre }
\end{aligned}
$$

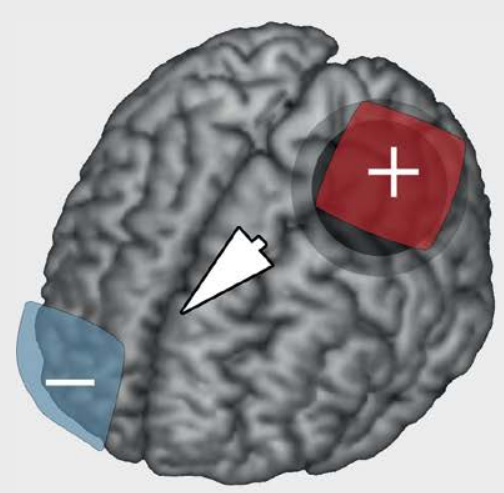

b

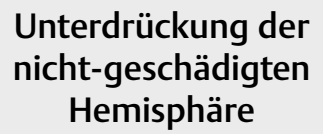

c Gleichzeitige Aktivierung der geschädigten und Unterdrückung der nicht-geschädigten Hemisphäre
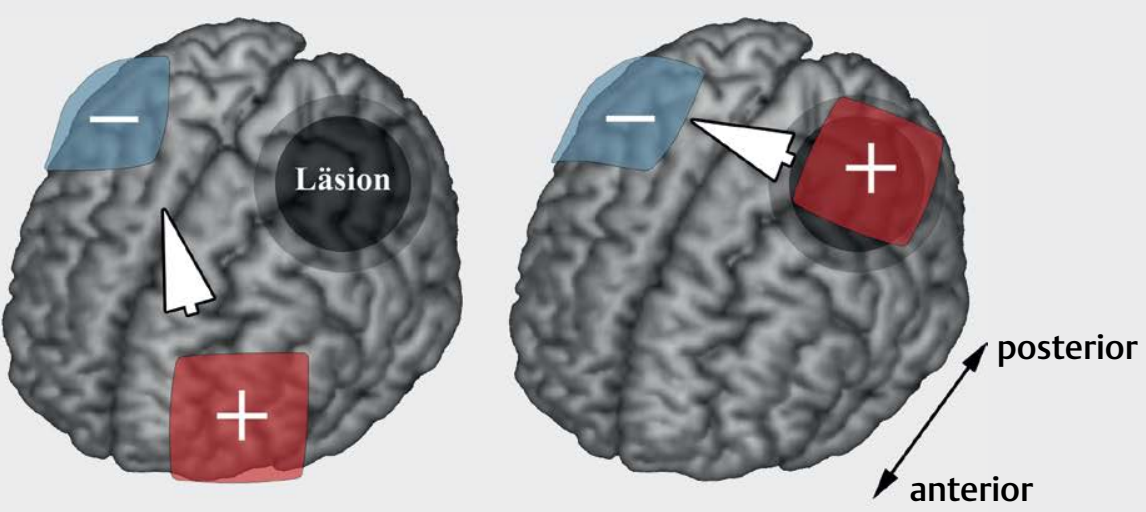

Abb. 2 Schematische Darstellung der 3 üblichen Hirnstimulations-Montagen am Beispiel der tDCS über dem Motorkortex. Hierbei wird entweder a die Anode (rot) über dem ipsiläsionellen Motorkortex plaziert und die Kathode (blau) auf der kontralateralen Stirn oder b die Kathode über dem Motorkortex der nicht-läsionellen Hemisphäre und die Anode auf der kontralateralen Stirn platziert. c Bei der bihemispärischen Montage wird die Anode über dem ipsiläsionellen Motorkortex und die Kathode über dem Motorkortex der nicht-läsionellen Hemisphäre platziert. Der weiße Pfeil symbolisiert die intrazerebrale Stromflussrichtung. Ziel der 3 Montagen ist es, die Interaktion zwischen beiden Motorkortizes durch eine Veränderung der Aktivität einer bzw. beider Hemisphären c zu modulieren.

besserung motorischer Fähigkeiten der oberen Extremität im Vergleich zu Scheinstimulation liegt dabei, je nach Studie und angewendetem Zielkriterium (z. B. Fugl-Meyer-Score, Wolf Motor Funktionstest, ARAT, Griffkraft, spezifische kinematische Parameter), im Bereich von 5 und 30\%. Neben der Heterogenität der Zielkriterien variiert in den Studien zusätzlich die Art der Intervention (motorisches Training, Physio/Ergotherapie, roboter-assistierte Therapie) und die Stimulation selbst (Zeitpunkt, Intensität, Dauer und Montage/Zielareal der tDCS). Die Patientenkollektive sind mit wenigen Ausnahmen klein und heterogen. Dementsprechend kommt eine aktuelle Cochrane Metaanalyse, obwohl frühe „proofof-principle“ Studien nicht eingeschlossen sind, zu dem Schluss, dass aufgrund der Studienheterogenität zum jetzigen Zeitpunkt bei geringer Qualität der Studienlage geringe Evidenz für eine Verbesserung von Aktivitäten des täglichen Lebens durch tDCS gegeben ist, jedoch Evidenz vorliegt für eine fehlende Verbesserung motorischer Defizite [23]. Wird gezielt nur die motorische Funktion der oberen Extremität als primäres Zielkriterium evaluiert, zeigen weitere Metaanalysen jedoch einen möglichen positiven Effekt der tDCS in Kombination mit Rehabilitationsprogrammen [24]. Wenn nur das spezifische motorische Training als Intervention berücksichtigt wird, findet sich sogar ein eindeutig positiver Effekt der tDCS auf motorische Funktion der oberen Extremität, im Vergleich zu Scheinstimulation oder Rehabilitationsinterventionen alleine [25]. Es sei angemerkt, dass die Ein- und Ausschlusskriterien sowie die primären Zielkriterien aller Metaanalysen differieren.

Zusammengefasst kann zum aktuellen Zeitpunkt ein vorteilhafter Effekt der tDCS auf die Verbesserung der Alltagsaktivitäten und der motorischen Funktion nach Schlaganfall angenommen werden. Um diese Annahme zu untermauern sind jedoch 2 wesentliche Aspekte zukünftig zu adressieren: 1. Das Zeitfenster nach Schlaganfall, 2. die Studienheterogenität. Die o.g. Metaanalysen basieren 
auf Daten, die zum Großteil in Patienten im chronischen Stadium (3 Monate bis Jahre) nach Schlaganfall erhoben wurden. Es fehlen Studien zu den Effekten der tDCS bei Patienten in der Akutphase nach Schlaganfall. Wie in $\mathbf{A}$ Abb. 1 ersichtlich sind bislang nur 2 Studien an Akutpatienten publiziert [26, 27]. In beiden Arbeiten wurde die tDCS ohne simultane Therapie/Training verabreicht und war im Vergleich zu einer Scheinstimulation uneffektiv. Aufgrund des in der Akutphase nach Schlaganfall vorliegenden plastischen Milieus könnte die gezielte Kombination von tDCS und Therapie hier vielversprechende Effekte auf den Rehabilitationsverlauf bewirken oder aber die „plastische Phase“ verlängern. Laufende Studien befassen sich genau mit diesem Aspekt, darunter unsere eigenen Forschungsarbeiten (Emmy Noether Programm „Mechanisms and Modulation of Motor Skill learning in the healthy and injured Brain“) sowie eine großangelegte, multizentrische Studie (DFG, NETS TRIAL: Neuroregeneration enhanced by tDCS in stroke, Studienkoordination: C. Gerloff, Hamburg). Weitere kontrollierte, randomisierte, prospektive, multizentrische Studien im klinischen Kontext sind nötig um die Wertigkeit der tDCS und des Timings in der Rehabilitation motorischer Funktion nach Schlaganfall zu untermauern. Darüber hinaus muss die Evaluation der Interaktion von tDCS und krankheits-assoziierter neuroplastischer Veränderungen durch grundlagenwissenschaftliche Arbeiten unterstützt werden. Aktuell sind 5 tierexperimentelle Studien zur (t)DCS nach akutem Schlaganfall veröffentlicht [28-32]. Ein gemeinsames Merkmal aller Arbeiten ist die (wiederholte) (t)DCS unter Anästhesie. Interaktionseffekte zwischen beiden Modalitäten sind nicht auszuschließen, insbesondere da beide auf die Erregbarkeit von Neuronen wirken. Eine Kombination der tDCS mit einem Training oder „Rehabilitation“ ist bisher nicht getestet worden, was somit der isolierten Beurteilung der Modulation der spontanen biologischen Erholung gleich kommt. Die verwendeten Stromdichten liegen deutlich oberhalb der humanen $0,8 \mathrm{~A} / \mathrm{m}^{2}$, z. T. sogar oberhalb der Läsionsschwelle für die anodale tDCS im gesunden Nager [2]. Darunter ergaben sich tendenziell neurodegenerative Effekte für die anodale Stimulation und z. T. positive Effekte für die kathodale Stimulation in Bezug auf Verhalten und Infarktvolumen [30-32]. In den 2 Studien mit der geringsten Stromdichte ( 1 bzw. $28 \mathrm{~A} / \mathrm{m}^{2}$, beide transkutan) beeinflusste die anodale tDCS das kognitive und motorische Verhalten der Nager positiv, ohne das Infarktvolumen zu verändern [28, 29]. Für die erfolgreiche Translation in den klinischen Kontext und das mechanistische Verständnis im erkrankten Gehirn (Interaktion von Pathologie und Stimulationseffekt) sind auch im grundlagenwissenschaftlichen Bereich weitere Studien notwendig, deren Design die Anwendung im wachen Tier und Stimulationsparameter in Anlehnung an die Humanbedingung beinhalten sollte.

\section{Aphasie und Neglekt nach Schlaganfall}

Auch Störungen nichtmotorischer kognitiver Funktionen wie Sprache und Raumwahrnehmung sind nach einem Schlaganfall häufig. In Analogie zum Hemisphärenkonzept bei motorischen Störungen findet sich eine ähnliche Rationale für die Anwendung der tDCS bei Neglekt oder Aphasie: die gezielte Modulation des betroffenen Hirnareals oder umliegender periläsioneller Areale einerseits und die Modulation der intakten Hemisphäre andererseits. Die Datenlage bei Neglekt ist zum jetzigen Zeitpunkt relativ gering. Im Gegensatz zu den motorischen Störungen liegen bislang nur „proof-of-concept“ Studien vor, bei denen die tDCS überwiegend allein, d. h. ohne z. B. simultanes Wahrnehmungstraining, und einmalig appliziert wurde. Wiederholte Sitzungen wurden bisher nur in 3 Studien verwendet [33-35], wobei in ersterer ein Feedbacktraining erfolgte und in zweiterer eine Kombination mit Prismenadaptation als Training verwendet wurde. Sowohl die „proofof-concept“ Studien als auch die Studien mit wiederholter Applikation belegen eine Verminderung der Neglekt-Symptome in spezifischen Tests (Linienhalbierung, Such- und Durchstreichaufgaben) nach anodaler Stimulation des rechten, d. h. ipsiläsionellen posterioren parietalen Kortex oder kathodaler Stimulation des linken, nicht-betroffenen posterioren parietalen Kortex im Vergleich zu einer Scheinstimulation. Interessanterweise findet sich für die synchrone bihemisphärische tDCS beider posteriorer parietaler Kortizes ein variables Studienergebnis [34, 35], was möglicherweise auf eine intraindividuell unterschiedliche Rolle beider Hemisphären in der Funktionserholung bei Neglekt hindeuten könnte. Metaanalysen liegen bislang nicht vor.

Sprachstörungen bedeuten für die Betroffenen oftmals eine schwerwiegende Einschränkung der Kommunikation und Interaktion mit der Umwelt. Daher ergibt sich ein großes klinisches Interesse, die tDCS als adjuvante Therapie zur Behandlung von Sprachstörungen nach Schlaganfall zu evaluieren, wie sich an einer Vielzahl von Publikationen zeigt (siehe Studiendatenbank in [14]). Mit wenigen Ausnahmen wurden die Effekte der tDCS in Kombination mit Sprachtherapie oder Konversationstraining evaluiert und zeigen zusammengefasst positive Effekte bei Patienten im chronischen Stadium, während im subakuten Stadium variable Effekte beschrieben sind [36-38]. Das Stimulationsziel bei Aphasie ist deutlich heterogener (siehe [37]; positive Effekte der anodalen tDCS wurden in einzelnen Arbeiten für den Gyrus frontalis inferior und den posterioren Gyrus temporalis superior der geschädigten Hemisphäre berichtet. Kathodale tDCS über dem Gyrus frontalis inferior der nichtgeschädigten Hemisphäre ergab ähnliche positive Effekte. Interessanterweise zeigt aber auch die Stimulation des linken Motorkortex, welcher anatomisch weiter entfernt von der strukturellen Läsion der primären Sprachareale liegt, die Kommunikationsfähigkeit zu verbessern, was durch eine verbesserte Interaktion mit dem residuellen, individuell unterschiedlichen Sprachnetzwerk erklärt werden könnte [39]. Primäre Zielparameter waren überwiegend Benennfähigkeit, Wortflüssigkeit und Wortverständnis. Eine Metaanalyse mit dem primären Zielkriterium Benennfähigkeit ergab einen signifikanten Vorteil der tDCS gegenüber Kontrollbedingungen, allerdings nur bei Patienten im chronischen Stadium nach Schlaganfall [40]. Eine weitere Metaanalyse, in der das primäre Zielkriterium „Kommunikationsfähigkeit“ aufgrund mangelnder Datenlage nicht beurteilt werden konnte, ergab für die Benennfähigkeit als sekundäres Zielkriterium über 6 Studien berechnet allerdings bei ähnlicher gemittelter Standarddifferenz keinen signifikanten Effekt der tDCS in Kombination mit Sprachtherapie gegenüber Scheinstimulation und Sprachtherapie [41]. Allerdings wurde in letzter Metaanalyse nicht zwischen den verschiedenen Phasen nach Schlaganfall unterschieden. 


\section{Bewusstsein und Aufmerksamkeit}

Störungen von Aufmerksamkeit und Wachheit, z. B. nach traumatischer Hirnverletzung, können einen erheblichen Einfluss auf den Rehabilitationsverlauf nehmen. Die Anwendung transkranieller Hirnstimulation wurde diesbezüglich nur in kleinen Fallberichten und offenen klinischen Studien exploriert. Bei Patienten im Zustand minimalen Bewusstseins (minimally conscious state [42]) erzielte die anodale tDCS über dem linken dorsolateralen präfrontalen Kortex kurzanhaltende Verbesserungen von klinisch messbaren Reaktionen, Hirnstammreflexen und Arousal, während Patienten mit apallischem Syndrom nicht profitierten [43]. Bei Patienten mit traumatischer Hirnverletzung und chronisch anhaltenden Defiziten von Gedächtnis und Aufmerksamkeit ließen sich für die einzelne wie wiederholte Stimulation vor kognitivem Training gemischte Effekte der tDCS über dem DLPFC nachweisen. Während Lesniak und Kollegen [44] keinen Effekt der tDCS fanden, sind positive Effekte der tDCS vor kognitivem Training auf spezifische kognitive Subdomänen wie z. B. die geteilte Aufmerksamkeit, Wortinterferenz, und Impulskontrolle berichtet worden $[45,46]$.

\section{Chronische motorische und nicht- motorische Symptome bei M. Parkinson}

Neben den vielfältigen Schlaganfallstudien, in denen mit tDCS funktionell in das sensomotorische oder attentionale System eingegriffen wird, ergeben sich ähnliche Fragestellungen auch bei neurodegenerativen Erkrankungen, die mit motorischen und nichtmotorischen Symptomen einhergehen wie bspw. dem Morbus Parkinson. Zum jetzigen Zeitpunkt ist die Studienlage jedoch gering. Untersuchungen bei Patienten mit idiopathischem Parkinsonsyndrom lassen einen günstigen Effekt der tDCS auf spezifische Symptome, z. B. Verbesserung von Arbeitsgedächtnis nach Stimulation des dorsolateralen präfrontalen Kortex [47, 48], oder eine Reduktion von Dyskinesien nach anodaler Stimulation von Motorkortex oder Cerebellum erkennen [49]. Bei Erkrankung mit Störung der Kortex-Basalganglien-Achse gibt es neuere wissenschaftliche Ansätze auch die Wechselstromstimulation (tACS) oder eine Wechselstrom-Stimulation mit randomisiert variierten Frequenzen zwischen 100 und $640 \mathrm{~Hz}$ (transcranial random noise stimulation, tRNS) einzusetzen. Die Rationale ist hierbei die Beeinflussung intrinsischer kortikaler und subkortikaler Oszillationen (im Sinne eines „Entrainments“ oder einer Phasenauslöschung, [50, 51]). Erste Ergebnisse bei tremordominantem Morbus Parkinson zeigen eine 50 \%ige Reduktion der Ruhetremor-Amplitude unter tACS [50]. Die 2 einzigen grundlagenwissenschaftlichen Arbeiten zu tDCS in Parkinson Nagermodellen zeigen positive Effekte der anodalen Stimulation auf behaviorale Parameter (partielle Normalisierung der Seitenpräferenz [52]; längere Laufzeiten im Rotarod-Test [53]). Mechanistisch zeigten sich die Dopamin- und Tyrosinhydroxylasekonzentration im gesamten Gehirn im Vergleich zu einer Scheinstimulation erhöht und die Konzentration oxidativer Stressparameter reduziert, was potenziell neuroprotektive Effekte der anodalen tDCS vermuten lässt. Kombinationen mit „Rehabilitation" oder Training sind bislang nicht untersucht. Weitere multimodale Untersuchungen zu potenziell neuroprotektiven Effekten der tDCS in einem „dose-response“ Design oder zumindest nahe den human angewandten Stimulationsstärken sind für die rationale klinische Anwendung wichtig.

\section{Sicherheitsüberlegungen}

Für die tDCS kann auf eine große Anzahl von Studieneinheiten zur Beurteilung der Sicherheit zurückgegriffen werden. Bis heute sind mehr als 1000 Probanden und Patienten in über 33200 Sitzungen mit tDCS (unter Verwendung o.g. konventioneller Stimulationsprotokolle) untersucht bzw. behandelt worden, ohne dass schwerwiegende Nebenwirkungen (serious adverse events) berichtet wurden [1]. Typische zu erwartende Nebenwirkungen aller nichtinvasiver elektrischer Hirnstimulationsverfahren sind Jucken und Brennen auf der Kopfhaut, Kopfschmerzen, Hautrötung im Bereich der Stimulationselektroden und generelles Unwohlsein, welche jedoch oftmals auch unter Scheinstimulation berichtet werden $[1,54]$. Aktuell gibt es keine Berichte über die Auslösung epileptischer Anfälle und auch die tierexperimentellen Daten aus Epilepsiemodellen lassen für die tDCS selbst in einem epileptischen Gehirn kein erhöhtes Risiko für eine Anfallsauslösung erkennen [1]. Es bleibt anzumerken, dass die physiologischen und behavioralen Effekte der tDCS bei traumatischer Hirnverletzung, insbesondere bei Vorliegen von Kalottendefekten, möglicherweise variabler sind, da Änderungen der intrakraniellen Stromflussrichtung möglich erscheinen und somit andere Hirnregionen erreicht werden als angenommen wird. Gleiche Überlegungen gelten für zerebrale Läsionen. Aktuelle Simulationen berechnen für Patienten mit Kalottendefekten eine bis zu 6-fach höhere intrazerebrale Stromdichte, was jedoch für die derzeitig angewendeten Stimulationsparameter (bis zu 0,8 A/m2) kein generelles Sicherheitsrisiko und somit kein Ausschlusskriterium von Patienten darstellt [1].

\section{FAZIT FÜR DIE PRAXIS}

Für eine Vielzahl neurologischer Symptome, die im Fokus der Neurorehabilitation stehen, sind positive Effekte der tDCS berichtet worden. Hervorzuheben sind die langfristige Verbesserung von Alltagskompetenzen, die Reduktion von Neglekt in der subakuten Phase und die Reduktion der Aphasie in der chronischen Phase nach Schlaganfall. Für eine informierte klinische Anwendung der tDCS in der Neurorehabilitation sind zukünftig auch Untersuchungen an Akutpatienten sowie grundlegende mechanistische Arbeiten notwendig.

Interessenkonflikt

Die Autoren geben an, dass kein Interessenkonflikt besteht.

\section{Englische Version}

Dieser Beitrag wurde auf Englisch publiziert in Neurology International Open 2017; 1: E142-E147 
Literatur

[1] Bikson M, Grossman P, Thomas C et al. Safety of transcranial direct current stimulation: evidence based update 2016. Brain Stimul 2016; 9: 641-661, DOI: 10.1016/j.brs.2016.06.004

[2] Gellner A-K, Reis J, Fritsch B. Glia: A neglected player in non-invasive direct current brain stimulation. Front Cell Neurosci 2016; 10: 188

[3] Bindman LJ, Lippold OC, Redfearn JW. Long-lasting changes in the level of the electrical activity of the cerebral cortex produced bypolarizing currents. Nature 1962; 196: 584-585

[4] Gartside IB. Mechanisms of sustained increases of firing rate of neurones in the rat cerebral cortex after polarization: role of protein synthesis. Nature 1968; 220: 382-383

[5] Nitsche M, Paulus W. Excitability changes induced in the human motor cortex by weak transcranial direct current stimulation. J Physiol 2000; 527 (Pt 3): 633-639

[6] Nitsche MA, Paulus W. Sustained excitability elevations induced by transcranial DC motor cortex stimulation in humans. Neurology 2001; 57: 1899-1901

[7] Fritsch B, Reis ], Martinowich K et al. Direct current stimulation promotes BDNF-dependent synaptic plasticity: potential implications for motor learning. Neuron 2010; 66: 198-204

[8] Podda MV, Cocco S, Mastrodonato A et al. Anodal transcranial direct current stimulation boosts synaptic plasticity and memory in mice via epigenetic regulation of Bdnf expression. Sci Rep 2016; 6: 22180

[9] Ranieri F, Podda MV, Riccardi E et al. Modulation of LTP at rat hippocampal CA3-CA1 synapses by direct current stimulation. J Neurophysiol 2012; 107: 1868-1880

[10] Reis ], Schambra HM, Cohen LG et al. Noninvasive cortical stimulation enhances motor skill acquisition over multiple days through an effect on consolidation. Proc Natl Acad Sci U S A 2009; 106: 1590-1595

[11] Buch ER, Santarnecchi E, Antal A et al. Effects of tDCS on motor learning and memory formation: a consensus and critical position paper. bioRxiv 2016; Available at http://biorxiv.org/content/ early/2016/07/18/064204.abstract

[12] Lefaucheur J-P, Antal A, Ayache SS et al. Evidence-based guidelines on the therapeutic use of transcranial direct current stimulation (tDCS). Clin Neurophysiol 2016; 128: 56-92

[13] Cappon D, Jahanshahi M, Bisiacchi P. Value and efficacy of transcranial direct current stimulation in the cognitive rehabilitation: a critical review since 2000. Front Neurosci 2016; 10: 157

[14] Lefaucheur J-P. A comprehensive database of published tDCS clinical trials (2005-2016). Clin Neurophysiol 2016; 46: 319-398, DOI:10.1016/j.neucli.2016.10.002

[15] Verheyden G, Nieuwboer A, Wit LD et al. Time course of trunk, arm, leg, and functional recovery after ischemic stroke. Neurorehabil Neural Repair 2007; Available at http://www.ncbi.nlm.nih.gov/entrez/query.fc gi? cmd = Retrieve \&db = PubMed\&dopt = Citation\&list_uids $=17876069$

[16] Kwakkel G, Kollen B, Twisk J. Impact of time on improvement of outcome after stroke. Stroke 2006; 37: 2348-2353

[17] Flowers HL, Skoretz SA, Silver FL et al. Poststroke aphasia frequency, recovery, and outcomes: a systematic review and meta-analysis. Arch Phys Med Rehabil 2016; 97: 2188-2201.e8

[18] Grefkes C, Ward NS. Cortical reorganization after stroke: how much and how functional? Neuroscientist 2014; 20: 56-70

[19] Duque J, Hummel F, Celnik P et al. Transcallosal inhibition in chronic subcortical stroke. Neuroimage 2005; 28: 940-946

[20] Murase N, Duque J, Mazzocchio R et al. Influence of interhemispheric interactions on motor function in chronic stroke. Ann Neurol 2004; 55 : 400-409
[21] Allman C, Amadi U, Winkler AM et al. Ipsilesional anodal tDCS enhances the functional benefits of rehabilitation in patients after stroke. Sci Transl Med 2016; 8: 330re1

[22] Mortensen J, Figlewski K, Andersen H. Combined transcranial direct current stimulation and home-based occupational therapy for upper limb motor impairment following intracerebral hemorrhage: a double-blind randomized controlled trial. Disabil Rehabil 2016; 38: 637-643

[23] Elsner B, Kugler J, Pohl M et al. Transcranial direct current stimulation (tDCS) for improving activities of daily living, and physical and cognitive functioning, in people after stroke. Cochrane Database Syst Rev 2016; 3: CD009645

[24] Tedesco Triccas L, Burridge JH, Hughes AM et al. Multiple sessions of transcranial direct current stimulation and upper extremity rehabilitation in stroke: A review and meta-analysis. Clin Neurophysiol 2016; 127: 946-955

[25] Kang N, Summers J], Cauraugh JH. Transcranial direct current stimulation facilitates motor learning post-stroke: a systematic review and meta-analysis. J Neurol Neurosurg Psychiatry 2016; 87: 345-355

[26] Rossi C, Sallustio F, Di Legge $S$ et al. Transcranial direct current stimulation of the affected hemisphere does not accelerate recovery of acute stroke patients. Eur J Neurol 2013; 20: 202-204

[27] Di Lazzaro V, Dileone M, Capone F et al. Immediate and late modulation of interhemipheric imbalance with bilateral transcranial direct current stimulation in acute stroke. Brain Stimul 2014; 7: $841-848$

[28] Kim SJ, Kim BK, Ko YJ et al. Functional and histologic changes after repeated transcranial direct current stimulation in rat stroke model. J Korean Med Sci 2010; 25: 1499-1505

[29] Yoon K], Oh B-M, Kim D-Y. Functional improvement and neuroplastic effects of anodal transcranial direct current stimulation (tDCS) delivered 1 day vs. 1 week after cerebral ischemia in rats. Brain Res 2012; 1452: 61-72

[30] Peruzzotti-Jametti L, Cambiaghi M, Bacigaluppi M et al. Safety and efficacy of transcranial direct current stimulation in acute experimental ischemic stroke. Stroke 2013; 44: 3166-3174

[31] Notturno F, Pace M, Zappasodi F et al. Neuroprotective effect of cathodal transcranial direct current stimulation in a rat stroke model. J Neurol Sci 2014; 342: 146-151

[32] Braun R, Klein R, Walter HL et al. Transcranial direct current stimulation accelerates recovery of function, induces neurogenesis and recruits oligodendrocyte precursors in a rat model of stroke. Exp Neurol 2016; 279: 127-136

[33] Yi YG, Chun MH, Do KH et al. The effect of transcranial direct current stimulation on neglect syndrome in stroke patients. Ann Rehabil Med 2016; 40: 223-229

[34] Làdavas E, Giulietti S, Avenanti A et al. a-tDCS on the ipsilesional parietal cortex boosts the effects of prism adaptation treatment in neglect. Restor Neurol Neurosci 2015; 33: 647-662

[35] Bang D-H, Bong S-Y. Effect of combination of transcranial direct current stimulation and feedback training on visuospatial neglect in patients with subacute stroke: a pilot randomized controlled trial. J Phys Ther Sci 2015; 27: 2759-2761

[36] Torres J, Drebing D, Hamilton R. TMS and tDCS in post-stroke aphasia: Integrating novel treatment approaches with mechanisms of plasticity. Restor Neurol Neurosci 2013; 31: 501-515

[37] Monti A, Ferrucci R, Fumagalli M et al. Transcranial direct current stimulation (tDCS) and language. J Neurol Neurosurg Psychiatry 2013; 84: $832-742$

[38] Hamilton RH, Chrysikou EG, Coslett B. Mechanisms of aphasia recovery after stroke and the role of noninvasive brain stimulation. Brain Lang 2011; 118: 40-50 
[39] Meinzer M, Darkow R, Lindenberg R et al. Electrical stimulation of the motor cortex enhances treatment outcome in post-stroke aphasia. Brain 2016; 139: 1152-1163

[40] Shah-Basak PP, Wurzman R, Purcell JB et al. Fields or flows? A comparative metaanalysis of transcranial magnetic and direct current stimulation to treat post-stroke aphasia. Restor Neurol Neurosci 2016; 34: 537-558

[41] Elsner B, Kugler J, Pohl M et al. Transcranial direct current stimulation (tDCS) for improving aphasia in patients with aphasia after stroke. Cochrane database Syst Rev 2015; CD009760

[42] Giacino JT, Ashwal S, Childs $N$ et al. The minimally conscious state: definition and diagnostic criteria. Neurology 2002; 58: 349-353

[43] Thibaut A, Bruno M-A, Ledoux D et al. tDCS in patients with disorders of consciousness: sham-controlled randomized double-blind study. Neurology 2014; 82: 1112-1118

[44] Leśniak M, Polanowska K, Seniów J et al. Effects of repeated anodal tDCS coupled with cognitive training for patients with severe traumatic brain injury: a pilot randomized controlled trial. J Head Trauma Rehabil 2014; 29: E20-E29

[45] Ulam F, Shelton C, Richards L et al. Cumulative effects of transcranial direct current stimulation on EEG oscillations and attention/working memory during subacute neurorehabilitation of traumatic brain injury. Clin Neurophysiol 2015; 126: 486-496

[46] Sacco K, Galetto V, Dimitri D et al. Concomitant use of transcranial direct current stimulation and computer-assisted training for the rehabilitation of attention in traumatic brain injured patients: behavioral and neuroimaging results. Front Behav Neurosci 2016; 10: 57
[47] Doruk D, Gray Z, Bravo GL et al. Effects of tDCS on executive function in Parkinson's disease. Neurosci Lett 2014; 582: 27-31

[48] Manenti R, Brambilla M, Benussi A et al. Mild cognitive impairment in Parkinson's disease is improved by transcranial direct current stimulation combined with physical therapy. Mov Disord 2016; 31: 715-724

[49] Ferrucci R, Cortese F, Bianchi M et al. Cerebellar and motor cortical transcranial stimulation decrease levodopa-induced dyskinesias in parkinson's disease. Cerebellum 2016; 15: 43-47

[50] Brittain J-S, Probert-Smith P, Aziz TZ et al. Tremor suppression by rhythmic transcranial current stimulation. Curr Biol 2013; 23: 436-440

[51] Vosskuhl J, Strüber D, Herrmann CS. Transcranial alternating current stimulation. Entrainment and function control of neuronal networks. Nervenarzt 2015; 86: 1516-1522

[52] Li Y, Tian X, Qian L et al. Anodal transcranial direct current stimulation relieves the unilateral bias of a rat model of Parkinson's disease. Conf Proc Annu Int Conf IEEE Eng Med Biol Soc IEEE Eng Med Biol Soc Annu Conf 2011; 2011: 765-768

[53] Lu C, Wei Y, Hu R et al. Transcranial direct current stimulation ameliorates behavioral deficits and reduces oxidative stress in 1-methyl-4-phenyl-1,2,3,6-tetrahydropyridine-induced mouse model of Parkinson's disease. Neuromodulation 2015; 18: 442-446 discussion 447

[54] Brunoni AR, Amadera J, Berbel B et al. A systematic review on reporting and assessment of adverse effects associated with transcranial direct current stimulation. Int J Neuropsychopharmacol 2011; 14: 1133-1145 